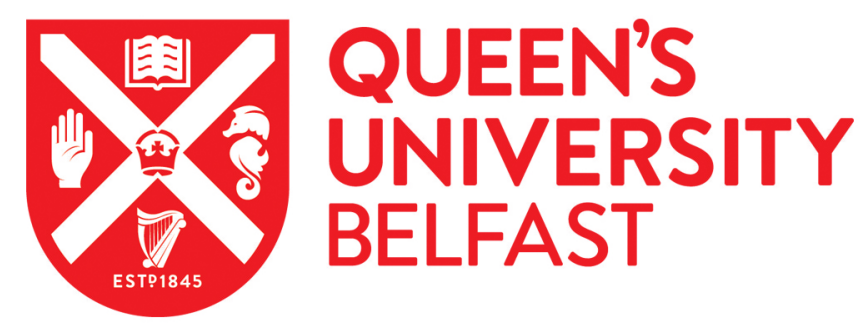

\title{
System constraints on efficacious teacher behaviours in school-based suicide prevention initiatives; a qualitative study of teacher views and experiences
}

McConnellogue, S., \& Storey, L. (2017). System constraints on efficacious teacher behaviours in school-based suicide prevention initiatives; a qualitative study of teacher views and experiences. Emotional \& Behavioural Difficulties, 22(2), 174-183. https://doi.org/10.1080/13632752.2017.1290897

\section{Published in:}

Emotional \& Behavioural Difficulties

\section{Document Version:}

Peer reviewed version

Queen's University Belfast - Research Portal:

Link to publication record in Queen's University Belfast Research Portal

\author{
Publisher rights \\ (C) 2017 SEBDA. \\ This work is made available online in accordance with the publisher's policies. Please refer to any applicable terms of use of the publisher.
}

\section{General rights}

Copyright for the publications made accessible via the Queen's University Belfast Research Portal is retained by the author(s) and / or other copyright owners and it is a condition of accessing these publications that users recognise and abide by the legal requirements associated with these rights.

Take down policy

The Research Portal is Queen's institutional repository that provides access to Queen's research output. Every effort has been made to ensure that content in the Research Portal does not infringe any person's rights, or applicable UK laws. If you discover content in the Research Portal that you believe breaches copyright or violates any law, please contact openaccess@qub.ac.uk. 


\begin{abstract}
International concern about youth suicide has led to a growing consensus that schools are a potentially important location for suicide prevention efforts. The present study investigated the experiences and perceptions of teachers regarding a role in youth suicide prevention through semi-structured interviews which were analysed using Interpretative Phenomenological Analysis (IPA). Three themes are reported which contribute to an understanding of the construction of teacher efficacy in relation to a role in suicide prevention: baseline mastery, threats to efficacy and tension between personal and professional identities. The results indicate a mix of positive and negative experiences relating to personal, process and systemic factors. Positive elements were associated with the pastoral role. Negative experiences related to referral pathways and broader systems of care. Implications for the development of school-based suicide prevention programmes and for conceptualising processes of change in school systems are discussed

Key words: youth suicide prevention; gatekeeper training; teachers' self-efficacy; schoolbased suicide prevention
\end{abstract}




\section{Introduction}

Suicide is the second most common cause of death among adolescents and young adults globally (World Health Organisation 2001; Hawton, Saunders \& O'Connor 2012). Suicide rates have increased by $60 \%$ in the past 45 years with young people being at highest risk in a third of countries. Schools are increasingly being asked to play a role in suicide prevention (Miller, Eckert \& Mazza 2009). Research has demonstrated that teachers recognise that suicide prevention is an issue in schools and that training is needed (Freedenthal \& Breslin 2010; Westefeld, Jenks Kettman, Lovmo \& Hey 2007). This study concerns the role of teachers in youth suicide prevention in a post-primary school context.

Growing concern regarding youth suicide prevention has led to recognition of the need to have teaching staff in schools trained to respond appropriately. Although evidence is still somewhat limited, one of the most promising interventions for school-based suicide-prevention efforts is gatekeeper training (Robinson, Cox, Malone, Williamson, Baldwin, Fletcher \& O'Brien 2013). These are programmes designed to equip individuals with the necessary 'knowledge, attitudes and skills to identify students at risk of suicide, determine the levels of risk and make referrals where necessary' (Gould, Greenberg \& Shaffer 2003, 395). The skills which are targeted include active listening and questioning and the most common gatekeeper training programmes include Applied Suicide Intervention Skills Training and safeTALK (LivingWorks Education). Research into the role of teachers in schoolbased suicide prevention strategies has focused largely on the outcomes of existing gatekeeper training programmes: such research assumes objective, quantifiable outcomes including knowledge about suicide, attitudes to the role and a measure of personal effectiveness in carrying out the role. 
Existing research has shown that, whilst teacher knowledge and attitudes are improved by gatekeeper training, teacher behaviours are not significantly affected (Keller et al. 2009; Reis \& Cornell 2008; Wyman, Inman \& Guo 2008). For example, Wyman et al. (2008) examined teachers' self-reported behaviour with students, such as how often they had asked students about distress or depressed mood since gatekeeper training. They found many adults were unprepared to initiate an interaction and/or make a direct response to a youth's suicidality.

A second key finding from survey-based research on teachers' perceptions of school-based suicide prevention is that teacher efficacy varies according to role and experiences, self-efficacy being a person's belief in their ability to succeed in carrying out a course of action in a particular situation (Bandura 2001). For example, higher efficacy has been found to be linked to individuals previously having had a student reveal suicidal thoughts to them (King, Price, Telljohann \& Wahl 1999).

It has also been found that teachers' views about their potential effectiveness in a suicide-prevention role is constrained by contextual factors. The importance of being able to refer directly within a school context has been demonstrated (Freedenthal \& Breslin 2010; Nadeem, Kataoka, Chang, Vona, Wong \& Stein 2011; Westefield et al. 2007). Whereas in a study of health education teachers only a quarter felt that if they talked to parents it would reduce the risk of suicide (27\%) (King et al. 1999). Conversely, where staff are informed about and have access to an organized system with transparent processes for responding effectively to a student who is at risk, self-efficacy of staff has been found to be higher (Anderson 2004; King et al. 1999) and more effective responses have been demonstrated (Stein, Kataoka, Hamilton, Schultz, Ryan, Vona \& Wong 2010). However, it is known that in many contexts, there is a lack of readily available, responsive mental health 
services in a health and social care context (Bamford 2006; Paternite \& Johnson 2005; Tomlinson 2007; Teggart \& Linden 2006).

Other contextual factors may differ, such as protocols for communication between schools and families. Another is definitions of teaching role. Reis and Cornell (2008) hypothesised, for example, that reluctance to ask students about suicide may result from teachers' perceptions of gatekeeping behaviours being outside the remit of their professional role. However, studies with subject teachers in a US context may not reflect the pastoral care role that is carried out by most postprimary teachers in a UK setting, in addition to their subject specific role.

Research using constructs such as knowledge, attitudes and self-efficacy may not be able to capture differing perceptions of a role in suicide prevention, contingent on the resources and care pathways available to teaching staff. Survey-based research is also limited in the extent to which reflections, emotions and interpersonal aspects of the self can be understood (Leary \& Tangney 2002). Qualitative research has been used to investigate the complexities inherent in assuming a role in suicide prevention, including emotional and relational experiences (Lachal, Orri, Sibeoni, Moro \& Revah-Leavy 2015) and the need for this has been illustrated through previous research demonstrating the complexities of responding across sectors (Wei, Kutcher \& Le Blanc 2015).

The aim of the research reported in this paper is to explore the subjective experiences and perceptions of teachers in a school-based suicide prevention role in depth. To achieve this, Interpretative Phenomenological Analysis (IPA, Smith 1996) is used. The research questions in this study are, therefore: what are teachers' experiences of working with young people at risk of suicide and how do they view this role? Existing research has focused on the views of specialists, such as health 
education teachers, counsellors, school psychologists or management (Eckert, Miller, DuPaul \& Riley-Tillman 2003; King et al. 1999; Reiss \& Cornell 2008; Scherff, Eckert \& Miller, 2005). This research focuses on the practitioners who have most daily contact with students - general teachers - who are almost invariably also pastoral care teachers in the UK.

\section{Method}

The present study involved semi-structured interviews with teachers that were transcribed and analysed using IPA. The participants, four female and three male, were recruited from one school in order to situate the analysis of results in the educational, socio-economic and cultural context of that school. The school was a large single-gender secondary school in an urban part of Northern Ireland. Having gained ethical approval for the study from the School of Psychology, Queen's University, Belfast, and the consent of the school Principal, letters of invitation were issued to all teaching staff, leading to seven volunteers. In the course of the study some participants indicated that they had participated in training on suicide prevention, but data concerning such training was not routinely collected, nor was it a factor in recruitment for the study. The participants ranged in age from thirty two to early sixties and the experience of teaching ranged from ten to thirty nine years. Participants included a senior teacher with responsibility for pastoral care and child protection a head of year and five form teachers.

The interview schedule used open-ended questions covering perception of role in suicide prevention, interpersonal issues, interagency issues, training and support and meanings of suicide and suicide prevention. The interviews, which lasted between 40 minutes and 80 minutes, were recorded using a digital audio voice 
recorder and the data was transcribed verbatim. Names have been changed to protect the anonymity of participants.

Steps were taken to minimise any potential emotional harm to participants, given the sensitive and challenging topic. These included initial briefing sessions, to enable enhanced informed consent, careful monitoring of participants to gauge if they were affected by the interviews, the right to withdraw at any time and information about helplines had been sourced, should it have been necessary.

\section{Analysis}

The analytic approach adopted for the research was IPA (Smith, 1996). This form of qualitative analysis is designed for use with data captured through semistructured interviews. It allows for an analysis which respects the individual experience but also recognises patterns and similarities between participants across the data set.

The themes generated are directly sourced from the data but the interpretative element of IPA allows for the use of theory and educational and psychological models as part of the process of making sense of the participants' experiences.

The use of IPA was the most suitable option in terms of sample selection (this was an experientially homogeneous sample), data source (semi-structured interview) and also the nature of the research question being addressed (the occupational and emotional lived experience). Discourse Analysis (Potter \& Wetherell, 1988) is too epistemologically relativist for this research which sought to contribute to social policy debates. Thematic Analysis (Braun \& Clarke, 2006) would have provided a suitable alternative if this had been part of a mixed method programme or had been based on a different data collection approach (eg focus group). 
IPA, along with other qualitative approaches, requires the researcher to do more than describe and report the data. The interpretative process can be driven by the data, for example, the analysis of a negative case (a transcript which produces very different themes from the rest of the dataset) can provide an insight into the whole corpus. Alternatively, a knowledge of theory or information from a literature review may inform the process of analysis. In this case, the theme of self-efficacy was articulated directly by the participants, though not necessarily using that terminology, and this enabled the next phase of the analytic process to be informed by Bandura's self-efficacy framework (Bandura, 1997).

\section{Results}

The results of the analysis highlight three inter-related themes reflecting the subjective experiences of the participants regarding a role in suicide prevention in a school context: baseline mastery; threats to efficacy and tension between personal and professional identities.

\section{Baseline mastery}

Most of the participants described their ability to identify signs of distress and/or respond to direct disclosures with knowledge and confidence. A potent aspect of the transcripts was that the meaning of suicidal ideation and behaviour in young people was constructed in a relational context. All the participants appeared to assimilate a role in suicide prevention into an existing pastoral care role.

...they're not just here for us to fill their heads with information and send them off with their diploma at the end of the day, they're actually here as whole people who need to be nurtured and minded. (Fiona) 
Participants described how they made use of existing skills, such as positive regard and active listening, and existing relationships with students to identify difficulties, make a connection, facilitate disclosure, and negotiate pathways to care. This appeared to afford the participants a strong sense of agency and control over what might be a highly stressful aspect of their job.

He needed help of a psychiatric nature and it was important to give him confidence that, you know...we were supporting him, we were behind him, that I wouldn't tell him a lie, that he could trust me. (Carl)

All the participants also expressed the belief that dealing with a young person who presents with suicidal ideation requires management of physical risk, necessitating the breaking of confidentiality and a passing on of responsibility to parents.

...in terms of child protection...it is instilled into us as teachers that there comes a stage when you should no longer be dealing with this, you have to pass it on. (Ann)

This theme captures the conditions which give rise to a sense of mastery agency and control - inherent in the accounts of the participants. These include the school's pastoral care structures and the ethos and values of the school, which provide a rationale for the participants to play an active role in youth suicide prevention. Concomitant skills arising from a relational view of teaching dominated the accounts. A shared understanding of safeguarding protocols also contributed to a 
sense of mastery, in the form of breaking confidentiality and referring concerns on to another party.

\section{Threats to efficacy}

For many of the participants there was a sense of diminishing control and a loss of agency experienced when the needs of vulnerable students had been passed on to others outside the school context. Whilst the experiences of the participants after notifying others outside the school of a young person's needs were diverse, knowledge about, understanding of and communication with the person/s or agency/ies to whom a young person's needs are communicated impacted greatly on participants' perceived effectiveness. Strong feelings arose out of these experiences.

...so I phoned the mother to work,...um which was a very difficult conversation [smiling] - hi I've got a suicide letter from your son - um...the father then phoned me and said can you read out the letter and I read out the letter over the phone to the father and then I suggested that he would bring the young man to the doctors or to the hospital and that would be my best advice. The parent came up, took the child home and two hours later sent him back to school... [long pause]...and it has never been mentioned again...I think it was a disgrace. I think we've failed him. (Elaine)

Elaine's feelings of anger after passing on her concerns to a student's family appear to arise from being unable, ultimately, to help a student who directly asked for help. It was highly compelling that many of the participants in the current study 
experienced families as barriers to care pathways in similarly negative ways with associated feelings of powerlessness, and personal vulnerability.

...they won't allow the child to go into counselling...and he does appear to need some form of some kind of,...intervention at the moment, that's just one example of where because we need permission from the parents to put the child into counselling or to give him any additional help because of his age, em....we're kind of, our hands are tied. (Fiona)

Participants also related feelings of frustration and disbelief regarding the response of health and social care professionals after the school had identified difficulties.

I didn't think the doctor was...didn't act really urgently on it because [the student] was in school the next day.... I didn't think that was good enough, I thought he should have been referred right away because my own experience told me this kid is vulnerable and, eh......(Carl)

I couldn't believe this, I was angry, it was disclosed at that [social services chid protection] case conference that that kid went to two hospitals and still didn't see anyone and that kid needed help. (Ben)

Some of the participants reported how relationships with students could be changed after onward referral, partly as a result of protocols for maintaining confidentiality and lack of feedback from parents and professionals outside school, leading to feelings of powerlessness to help the young person once parents had been informed. 
...you know I feel awkward in front of the child now, it's uncomfortable for him to have him in my class...he's closed off...I still maintain that child is depressed and has never had any help, um...but I've no way of communicating that to the GP. (Elaine)

This theme encapsulates how wider systems, outside the school context, can threaten an existing mastery which the participants felt they possessed.

\section{Tension between personal and professional identities}

The final theme relates to how the participants manage such threats to mastery in their professional role. There is a sense that the processing of strong emotions reported in the second theme results in conflicted identities, whereby feeling limited as a professional effects individuals personally. This is consequently linked to participants' evaluation of their potential impact as agents for change in relation to suicide prevention.

Many of the participants reflected on the impact of legalistic thinking and procedures on their personal experiences of their role, feeling, for example, open to judgement.

You're probably aware there was a child died by suicide in this school...and you know at that time I know a lot of records were asked for. (Ann)

Elaine's use of the metaphor of a robot communicates a feeling of being dehumanised in her professional identity by forces originating outside the school. 
They think you're robots when you're teaching young people. (Elaine)

Such social transmission of external beliefs about their role may have contributed to the resistance of some of the participants to a role in suicide prevention.

At the end of the day I can't be their parent. (Gerry)

You know we're not psychiatrists. We...our role is to identify and pass on as quickly as possible. (Elaine)

It was equally apparent that previous negative experiences after onward referral appeared to give rise to potentially self-protective statements which functioned to actively demarcate the limits of perceived professional obligations.

I can't send that child home from school knowing that that child is at risk so...em...to be honest I'm not sure legally but I would be happier to tell the parent, to tell somebody who's responsible for the child. (Diane)

Many of the participants also used the importance of time and physical place to delineate such parameters of responsibility.

When children come through that door in the morning we're in charge of them and we're not just in charge of their education, it's their health and wellbeing and you know in a sense we are acting as the parents would do with the child 
at home...[Parents] have the responsibility to ensure the health and safety of their child outside of school. (Ben)

Through taking a stance to protect the self against perceived failure and external judgement, the participants recounted actively constructing the limits of professional and personal responsibility in relation to a role in suicide prevention.

\section{Discussion}

The results reflect findings in the existing literature that teachers feel they have an ethical and legal responsibility to recognise and respond to students at risk of suicide (Anderson 2004). In the current study empowerment in carrying out this role appeared to arise from interpersonal encounters with vulnerable students. Such a relational perspective on how teachers can respond to the needs of young people who are suicidal is largely absent from the international literature.

Whilst participants' accounts were strongly informed by positive appraisals of their ability to make a difference via their own actions, powerlessness and loss of control was associated with contact with parties outside of the school context. This disparity inherent in the participants' perceptions of their role can be interpreted in terms of the theoretical construct of self-efficacy. Existing training for teachers in suicide prevention typically aims to increase efficacy expectation, which is concerned with an individual's perception of whether they can carry out an action (Bandura, 1997). The in-depth reflections of the participants in this study suggest that external factors are perceived as having more of an influence than internal factors for some teachers in this role (Rotter 1966). The participants' explored what it meant to them that the outcome they desired, that a young person in distress would 
receive help, was contingent upon the action of others (Bandura, 1997) and often did not come about (Hayes, Hindle \& Withington 2007).

The negative emotional responses associated with this, which included guilt, anger, anxiety and a need to defend the self against judgement, served to undermine the confidence of participants and, in some cases, their willingness to play a role in suicide prevention. This contrasts with previous research findings that prior experience of having students express suicidal thoughts to professionals has been linked to increased self-belief about a role in suicide prevention (King et al. 1999).

These findings illustrate the need to manage the expectations of teachers being trained to play a role in suicide prevention. One of the ways to do this is to conceptualise the skills and knowledge that teachers need to develop as contingent on the system in which a suicide-prevention approach will operate (TschannenMoran \& Ho, 2001; Maddux \& Gosselin 2001; Parker, Hannah \& Topping 2006). For example, teachers will require "knowledge of how the system works and an appraisal of one's ability to manage the institutional requirements" (Bandura 1997, 66). Previous research has found that high efficacy scores were related to knowledge about and access to suitable intervention (King et al. 1999; Anderson 2004).

In the absence of the management of such expectations, as illustrated through the experiences of the participants in this study, conflicted and, consequently, defended identities emerged in the accounts. Participants appear to actively attempt to manage a threat to self-hood by articulating the limitations of their own personal responsibility. This can be illuminated by the theoretical concept of the psychological contract, which is 'a set of beliefs about what [both management and workers are] entitled to receive and obligated to give' (Morrison \& Robinson 1997, 1998). The participants' views reflect existing findings in the literature of 
occupational change that reduced commitment to engage in behavioural change can arise when employees perceive employers as not having fulfilled their role adequately (Coyle-Shapiro \& Kessler 2000). In this study it is those external to the school who are perceived as not fulfilling their role, reflecting the need to conceptualise suicide-prevention training in a multi-agency context.

\section{Implications}

Youth suicide prevention involves a system at the interface between schools, families and health professionals. In the current study the experience of participants is largely negative as, in their role as teachers, they are largely dependent on parents to negotiate and secure onward referral to professionals with the expertise to intervene in the case of suicidal ideation. Previous research exploring the implementation of school-based suicide prevention programmes has demonstrated that the role of teachers in making an onward referral is made easiest when 'there is a structured referral process and on-site services that are widely known to staff and can easily be accessed' (Nadeem et al 2011, 217). Problems can arise when generic gatekeeper training for school-based professionals alludes to referral routes which transpire to be lacking (McLean, Schinkel, Woodhouse, Pynonnen \& McBride 2007). Therefore, attending to the contextual fit of evidence-based training programmes for practitioners has the potential to ensure more positive outcomes for teachers in school-based suicide prevention strategies. At present, authorities are often limited to commissioning existing training programmes which are for lay individuals and which are not specific to children and young people.

Ideally, mental health care should be available, in some form, in a school context and/or mental health services should take direct referrals from schools (WHO, 2000; Rothi \& Leavey 2006a). In the absence of this, commissioning of 
gatekeeping training should take place concurrently with the auditing of existing provision and protocols in care pathways for young people at risk of suicide.

Gatekeeper training for school-based suicide prevention should include detailed information about how the system works including known barriers in care pathways such as waiting lists or the reluctance of some families to act when a young person is identified as at risk for suicide (Rothi, \& Leavey 2006b). Existing gatekeeper training programmes are universal and, therefore, not tailored to context in this way.

The findings of this research also suggest that it would be helpful for teachers if suicide prevention training programmes were designed to acknowledge the existing skills, knowledge and capabilities that teachers have, with reference to their pastoral care role.

\section{Limitations}

This study adopted a qualitative approach which focuses on experiential accounts. Qualitative research naturally leads to self-selectivity in achieving a sample of participants. In this study this resulted in a cohort of individuals who had worked with vulnerable young people, many suicidal. Many had voluntarily undertaken suicide prevention gatekeeper training and were informed about the topic. Almost all were highly committed to a role in youth suicide prevention. This article attempts to represent the experiences of this one set of participants. As a result, generalizability is not possible. However, the findings of studying the in-depth reflections of a small group of individuals in relation to this complex role can contribute to a better understanding of the issues involved in designing school-based suicide prevention strategies. 
Further research might look at the perspective of young people, parents and those professionals on the receiving end of referrals to explore issues arising in this research.

\section{Conclusion}

This study increases our depth of knowledge about the role of teachers in school-based suicide prevention and can enhance the insight of professionals who directly support schools and teachers in this regard, such as Educational Psychologists and Behaviour Support Professionals.

Findings emphasise a positive aspect of teachers' perceptions of their role in suicide prevention by illustrating the existing relational and communicative potential of pastoral care processes and structures, conceptualised here as baseline mastery, within schools. This is an important addition to the existing international literature.

Findings also highlight, however, an important discordance arising from the unpredictability of the wider system surrounding the child. By embedding the perceived agency of the participants in the current study in a systemic context we learn that the capacity of teachers to function as intentional agents in relation to youth suicide prevention is constrained not only by their own knowledge and skills, typically addressed in training, but also by emotional and systemic processes. Those who are indirectly involved in school-based suicide prevention can support initiatives by examining referral protocols and the availability of services for young people at risk of suicide.

\section{Acknowledgements}

The doctoral study on which this paper draws was funded by the Department of Education (Northern Ireland). 


\section{References}

Anderson, W. 2004. "Self-efficacy: Perceptions of high school teachers related to adolescents at risk for suicide". Phd dissertation.

The Bamford Review (NI). 2006. A Vision of a Comprehensive Child and Adolescent Mental Health Service.

Bandura, A. 1986. Social Foundations of Thought and Action: A Social Cognitive Theory. Englewood Cliffs, NJ: Prentice-Hall.

Bandura, A. 1997. Self-efficacy. The Exercise of Control. New York: W.H. Freeman and Company.

Bandura, A. 2001. "Social cognitive theory: An agentic perspective." Annual Review of Psychology, 52: 1-26.

Braun, V. and Clarke, V 2006. "Using thematic analysis in psychology". Qualitative research in psychology. $3,2$.

Coyle-Shapiro, J. and Kessler, I. 2000 "Consequences of the psychological contract for the employment relationship: A large scale survey". The Journal of Management Studies. 37: 904-930.

Eckert, T., Miller, D., DuPaul, G. and Riley-Tillman, T. 2003. “Adolescent suicide prevention: school psychologists' acceptability of school-based programmes". School Psychology Review. 32: 57-76.

Freedenthal, S. and Breslin, L. 2010. "High school Teachers' Experiences with suicidal students: A Descriptive Study". Journal of Loss and Trauma. 15: 8392.

Gould, M., Greenberg, T. and Shaffer, D. 2003. "Youth suicide risk and preventive interventions: A review of the past 10 years". Journal of the American Academy of Child and Adolescent Psychiatry. 42 
Hawton, K., Saunders, K. and O'Connor, R. 2012. "Self-harm and suicide in Adolescents". Lancet. 379: 2373-2382.

Hayes, B., Hindle, S. and Withington, P. 2007. "Strategies for Developing Positive Behaviour Management. Teacher Behaviour Outcomes and Attitudes to the Change Process". Educational Psychology in Practice. 23: 161- 176.

Keller. D., Schut, L., Puddy, R., Williams, L., Stephens, R., McKeon, R. and Lubell, K. 2009. "Tennessee lives count: Statewide gatekeeper training for youth suicide prevention". Professional Psychology.40: 126-133.

King, K., Price, J., Telljohann, S. and Wahl, J. 1999. "High school health teachers perceived self-efficacy in identifying students at risk for suicide". The Journal of School Health. 69: 202-207.

Lachal, J., Orri, M., Sibeoni, J., Moro, M. \& Revah-Leavy, A. 2015 "Metasynthesis of Youth Suicidal Behaviours: Perspectives of Youth, Parents and Healthcare Professionals". Plos ONE. 10 (5): 1-25.

Learey, M. and Tangney, J. 2002. (Eds.) Handbook of Self and Identity. New York, London: The Guilford Press.

LivingWorks [Retrieved December 13, 2016]; http://www.livingworks.net/. Maddux, J. and Gosselin, J. 2002. Self-efficacy. In M.Leary and J. Tangney (Eds.) Handbook of Self and Identity. New York, London: The Guilford Press.

McClean, J., Schinkel, M., Woodhouse, A., Pynonnen, A. and McBride, L. 2007. Evaluation of the Scottish Safetalk pilot. Unpublished.

Miller, D., Eckert, T. and Mazza, J. 2009. "Suicide prevention programs in the schools: A review and public health perspective". School Psychology Review. 38: $168-188$.

Morrison, E. and Robinson, S. 1997. "When employees feel betrayed: A model of 
how psychological contract violation develops". Academy of Management Review. 22: 226-256.

Nadeem, E., Kataoka, S., Chang, V., Vona, P., Wong, M. and Stein, B. 2011. "The Role of Teachers in School-Based Suicide Prevention: A Qualitative Study of School Staff Perspectives”. School Mental Health. 3: 209-221.

Parker, K., Hannah, E. and Topping, K. 2006. "Collective teacher efficacy, pupil attainment and socio-economic status in primary school”. Improving Schools. 9, 111-130.

Paternite, C. and Johnston, T. 2005. "Rationale and strategies for central involvement of educations in effective school-based mental health programs". Journal of Youth and Adolescence. 34: 41-49.

Potter, J. and Wetherell, M. 1988 Discourse analysis and the identification of interpretative repertoires. In Antaki, C. (Ed) Analysing everyday explanation: A casebook of methods. Thousand Oaks, CA, US: Sage Publications.

Reis, C. and Cornell, D. 2008. “An evaluation of suicide gatekeeper training for school counsellors and teachers". Professional School Counseling. 11: 386393.

Robinson, J., Cox, G., Malone, Al, Williamson, M., Baldwin, G., Fletcher, and O’Brien, M. 2013. “A systematic review of school-based interventions aimed at preventing, treating and responding to suicide-related behaviour in young people”. Crisis. 34 (3): 164-182.

Rothi, D. and Leavey, G. 2006a. "Mental Health Help-Seeking and Young People: A Review". Pastoral Care. September.

Rothi, D., Leavey, G., Chamba, R. and Best, R. 2006b. Identification and 
management of pupils with mental health difficulties. London: NASUWT.

Rotter, J. 1966. “Generalized expectancies for internal versus external control of Reinforcement”. Psychological Mongraphs: General and Applied. 80: 1-28.

Rousseau, D. 2001. "Schema, promise and mutuality: The building blocks of the psychological contract”. Journal of Organizational Psychology. 74: 511-541.

Scherff, A., Eckert, T. and Miller, D. 2005. "Youth suicide prevention: A survey of public school superintendents' acceptability of school-based programs". Suicide and Life-Threatening Behavior. 35: 154-169.

Smith, J. 1996. "Beyond the divide between cognition and discourse: Using interpretative phenomenological analysis in health psychology". Psychology and Health. 11: 261-271.

Smith, J., Flowers, P. and Larkin, M. 2009. Interpretative Phenomenological Analysis. Theory, Method and Research. London: SAGE.

Stein, B., Kataoka, S, Hamilton, A., Schultz, D., Ryan, G, Vona, P. and Wong, M. 2010. "School Personnel Perspectives on their School's Implementation of a School-Based Suicide Prevention Program". The Journal of Behavioural Health Services \& Research. 37 (3): 338-349

Stevens, R. 1996. The reflexive self: An experiential perspective. In R. Stevens (Ed.) Understanding the Self. London: SAGE.

Teggert, T. and Linden, M. 2006. Investigating service users' and carers' views of child and adolescent mental health services in Northern Ireland. Child Care in Practice. 12: 27-41.

Tomlinson, M. 2007. "Suicide and young people: The case of Northern Ireland". Child Care in Practice. 13: 435-443.

Touroni, E. and Coyle, A. 2002. "Decision-making in planned lesbian parenting: An 
interpretative phenomenological analysis". Journal of Community and Applied Social Pscyhology.12: 194-209.

Tschannen-Moran, M. and Woolfolk Hoy, A. 2001. "Teacher efficacy: Capturing an elusive construct". Teaching and Teacher Education. 17: 783-805.

World Health Organisation. 2001. World Health Report. Mental illness: New understanding, new hope. Geneva: WHO.

Wei, Y., Kutcher, S., LeBlanc, J. 2015. "Hot Idea or Hot Air: A Systematic Review of Evidence for Two Widely Marketed Youth Suicide Prevention Programs and Recommendations for Implementation”. Journal of the Canadian Academy of Child and Adolescent Psychiatry. 24 (1): 5-16.

Westefeld, J., Jenks Kettman, J., Lovmo, C. and Hey, C. 2007. "High School Suicide: Knowledge and Opinions of Teachers". Journal of Loss and Trauma. 12: 33-44.

Wyman, P., Inman, J. and Guo, J. 2008. "Randomized trial of a gatekeeper program for suicide prevention: 1-Year impact on secondary school staff'. Journal of Consulting and Clinical Psychology. 76, 104-115. 